\title{
Vibrationally excited populations from IR-multiphoton absorption. 1. Absorbed energy and reaction yield measurements $s^{a}$
}

\author{
Jean-Michel Zellweger \\ Department of Chemical Kinetics, Chemical Physics Laboratory, SRI International, Menlo Park, California \\ 94025 \\ Trevor C. Brown \\ Department of Chemical Engineering, University of Adelaide, Box 498, G.P.O., Adelaide, South Australia \\ 5001 \\ John R. Barker \\ Department of Atmospheric and Oceanic Science, Space Physics Laboratory, University of Michigan, Ann \\ Arbor, Michigan 48109-2143
}

(Received 31 July 1985; accepted 6 September 1985)

\begin{abstract}
The molecule 1,1,2-trifluroethane (TFX) was used in experiments to determine the population distribution of excited molecules produce by infrared multiphoton absorption induced by high power TEA CO $\mathrm{CO}_{2}$ lasers operating at $1079.85 \mathrm{~cm}^{-1}[9.6 \mu \mathrm{m} R$ (22) line]. Optoacoustic

measurements of absorbed laser power provided a measure of the mean energy of the population distribution, while very low pressure photolysis measurements of the collision-free decomposition yield gave infornation abont the tigh energy thill of the distribution. The experimental results were accurately simulatec using a Master Equation model that incorporated Quack's statisticaldynamical theory of infrared multiphoton absorption (cases $B$ and C), RRKN unimolecular reactions (thres clkannels), and colisional energy transfer. The computer skmulations included known TEE molecular properties and only four axdjustable parameters, which were very highly constrained in order to fit the experimental data. From the siwulations, we conclude that the optical coupling matrix elements are drumatically reduced in magnitude for energies above the reaction thresholds. This effect is symptomatic of the vibrational anharmonicity due to the presence of the reaction channels, even in molecules that have not yet reacted, resulting in vibrational frequency shifts of the absorption lines out of resonance with the laser line. This effect is expected to be present and observable in other highly vibrationally excited molecules.
\end{abstract}

\section{INTRODUCTION}

A little more than ten years ago, great interest was aroused by the first observations of chemical reactions in. duced by infrared lasers. ${ }^{1}$ The observations could only be explained if isolated molecules absorbed many infrared photons, leading to bond fission and molecular elimination reactions. Because not all irradiated molecules absorb the same number of photons, the ropulation distributions are produced with substantial energy dispersions.

Despite the broad population distributions, IR-multiphoton absorption has great potential as a useful laboratory tool because of two features: (1) many molecules absorb light from poweriul infrared (e.g., $\mathrm{CO}_{2}$ ) lasers and therefore the effect is general, and (2) almost all irradiated molecules are excited to relatively high vibrational energies. By varying laser power, wavelength, and other parameters, the average energyof the population can be controlled, much as the average energy can be controlled in thermal systems by changing the temperature. Until the population distributions are better characterized, however, the usefulness of IRMPA is limited, despite its potential as a laboratory tool.

Although hundreds of publications have described IRMPA and several excellent reviews have been published, ${ }^{1}$

\footnotetext{
"All of the experimental work was carried out at SRI International and was supported by the U.S. Army Research Office.

${ }^{b)}$ Address correspondence to this author.
}

the details of the population distributions in large molecules are still not well known, but only have been inferred from limited measuremen $\left\{\mathrm{s}^{2.3}\right.$ and from theoretical calculations. In this paper, two experimental techniques (VLP $\Phi$ measurement of decomposition yields ${ }^{4}$ and optoacoustic measurement of absorbed laser power ${ }^{5}$ were employed along with a Master Equation theoretical mode ${ }^{6}$ to obtain improved estimates of the population distributions produced by IRMPA. By using more than one experimental technique, the theoretical modeling is highly constrained, leading to reliable estimates for the population distributions. A third experimental technique, infrared fluorescence spectroscopy, was also applied in this study and it shows excellent consistency with the other techniques, but it has not been independently calibrated and so it is described separately in the following paper.

1,1,2-trifluorethane (TFE) was chosen for the experiments for several reasons:

(1) A vibrational assignment ${ }^{8}$ has been made and TFE has a strongly absorbing C-F stretch mode near $1076 \mathrm{~cm}^{-1}$, suitable for pumping with a $\mathrm{CO}_{2}$ laser.

(2) TFE has simple unimolecular reactions (HF molecuJar eliminations by three reaction pathways), which have already been thoroughlly investigated in thermal and chemical activation experiments. ${ }^{9}$ These molecular elimination reactions do not lead to secondary chemistry, simplifying interpretation of the results. 


$$
\begin{aligned}
\mathrm{CF}_{2} \mathrm{H}-\mathrm{CFH}_{2} & \rightarrow \mathrm{CF}_{2}=\mathrm{CH}_{2}+\mathrm{HF} \\
& \rightarrow \text { cis- or trans-CFH }=\mathrm{CFH}+\mathrm{HF} \\
& \rightarrow \mathrm{CF}-\mathrm{CFH} H_{2}+\mathrm{HF} \\
& \rightarrow \text { cis- or trans-CFH }=\mathrm{CFH}+\mathrm{HF} .
\end{aligned}
$$

(3) The internal and external rotations in TFE are relatively massive and they have low symmetry, producing a high density of optically accessible vibrational states, which enhances the efficiency of IRMPA. Moreover, the wide dispersion of the vibrational frequencies produces a "filled-in" density of states, with few regions of low state density, except at very low energy.

(4) The $\mathrm{C}-\mathrm{H}$ stretch mode frequencies are conveniently separated from the frequencies of other modes in TFE, reducing the spectroscopic resolution requirements in the infrared fluorescence experiments. ${ }^{7}$

(5) The bimolecular reactions of TFE with atoms and simple free radicals are expected to be straightforward and suitable for studies of the effects of vibrational energy on bimolecular reactivity.

The usefulness of TFE has been borne out in the experiments reported here. High IRMPD yields were obtained and the experimental observations were entirely in accord with the qualitative expectations. The results are highly consistent and they lead to better knowledge of the properties of highly vibrationally excited molecules, as described in the following sections.

\section{EXPERIMENTAL}

The VIP $\Phi$ technique for measurement of collision-free IR multiphoton decomposition (IRMPD) yields has been described elsewhere in detail ${ }^{4}$ except for a few modifications. Gases at low pressure ( $<20 \mathrm{~m}$ Torr) are irradiated in a $2 \mathrm{~cm}$ diam by $10 \mathrm{~cm}$ long Knudsen cell equipped with $\mathrm{KCl}$ windows. The gas escapes through an aperture at a rate dependent only on the mean molecular velocity, the cell volume, and the area of the aperture. In the present experiments, the escape aperture was fabricated from a Teflon-bore greaseless stopcock in order to have continuous control of the residence time. Since the stopcock could not be reproducibly reset to known aperture areas, 1 Torr Baratron capacitance manometer (model 223A) was used to monitor gas pressure in the cell directly. The residence time $\tau$ is given by ${ }^{10}$

$$
\tau=P V_{\text {cell }} N_{0}\left(R T F_{A}\right) \text {, }
$$

where $P$ is the observed pressure, $V_{\text {cell }}$ is the cell volume, $N_{0}$ is Avogadro's number, $R$ is the gas law constant, $T$ the temperature, and $F_{A}$ is the measured gas flow rate (measured by timing the rate of pressure increase in a calibrated volume); the escape rate constant is $\tau^{-1}$.

The composition of the gas escaping from the cell is monitored by a modulated molecular beam mass spectrometer, as described elsewhere." For this purpose, a Finnegan Spectrascan 400 quadrupole mass spectrometer was used with an SRI-built "peak picker" for selection of specific mass peaks. The output of the quadrupole was amplified with a PAR model 181 charge-sensitive preamplifier and the modulated signal was isolated from background with an Ith- aco model 391 lock-in amplifier, locked to the $200 \mathrm{~Hz}$ tuning-fork chopper (Bulova Time Products). For futher signal averaging, the output from the lock-in was captured and stored by a Nicolet model 1072 signal averager, which was interfaced with a DEC LSI $11 / 2$ computer for storage of the data on floppy disks and subsequent analysis.

Two $\mathrm{CO}_{2}$ TEA lasers were used in the experiments: a low repetition rate laser (Lumonics model K103), which can deliver $>5 \mathrm{~J} /$ pulse at $<0.25 \mathrm{~Hz}$, and a high repetition rate laser (Tachisto, model $555 \mathrm{G}$ ), which can deliver $\leqslant 1 \mathrm{~J} /$ pulse at up to $22 \mathrm{~Hz}$. For most experiments, the lasers were operated on the $9.6 \mu \mathrm{m} R(22)$ line $\left(1079.85 \mathrm{~cm}^{-1}\right)$ and, in many experiments, a three-power Gallilean telescope (Janos Technology) was used to concentrate the beam. Laser pulse energy was monitored continuously with a volume-absorbing calorimeter (Scientech, Inc.) connected to a strip-chart recorder. Under our operating conditions, the thyratron-triggered Tachisto laser showed pulse-to-pulse energy variations of about $\pm 5 \%$, while the older, spark-gap-triggered Lumonics showed variations of more than $\pm 20 \%$.

Laser fluence was determined by the measured laser pulse energy, divided by the laser beam cross-sectional area. When the telescope was not used, the laser beam cross section was defined by an iris diaphram positioned before the entrance window. When the beam was concentrated by the telescope, the area was determined by scanning a pinhole in a two-dimensional survey across the beam, and applying the $1 / e$ criterion. The areas so defined were very similar to the patterns produced on thermal printer paper, which was used for quick checks of beam area. Additional confidence in the printer-paper measurements was gained by measuring the beam area before, and after the telescope: within $\pm 10 \%$, the ratio of the beam areas was 1/9. Laser fiuence inside the cell was assumed to equal that measured at the cell entrance, because reflection losses from the entrance window are nearly compensated by back reflections from the exit window and the smant residual correction is much less than the estimated $\pm 30 \%$ uncertainty in the absolute fluence.

The laser fiuence was varied in crude steps by attenuation with polyethylene films; fine adjustments to the fluence were made by varying the high voltage settings on the laser. At high repetition rates, the polyethylene films were cooled. with a stream of compressed air to prevent their deformation and melting.

For both lasers, the far-field pattern had hot and coid zones that differed from the average fiuence by $20 \%$ to $30 \%$. These patterns depended on laser alignment and optical path from the laser to the cell, but they were somewhat reproducible, and their presence did not seem to affect the results. The overall intensity profile of the beam from each laser was approximately trapezoical, similar to the profile measured in earlier work. ${ }^{12}$

IRMPD yields were determined using two variations of the VIP $\Phi$ technique: single-shot accumulation, or fast-repetition quasi-steady-state depletion. The first method has been described previously. The quasi-steady-state depletion method used the fast repetition rate capability of the Tachisto laser to repeat laser shots many times during the gas residence time. Since each parcel of gas was irradiated many 
times prior to exiting the cell, larger depletions of reactant gas were generated, allowing measurement of smaller yieldsper-shot that could be attained using the single-shot accumulation technique. Tests showed that 1,1-difluoroethylene (the only ethylene reaction product commercially available: PCR, Inc.) does not absorb the laser photons. The reported vibrational frequencies of 1,1-difluoroethylene and of cisand trans-1,2-difluoroethylene (the other products) indicate that they will not absorb at the laser wavelength. ${ }^{13}$ (Yields were also measured optoacoustically, as described below.)

In the absorbed laser energy experiments, the cell was a Pyrex cylinder $(4 \mathrm{~cm}$ diam $\times 30 \mathrm{~cm}$ long) equipped with inlet and outlet stopcocks to facilitate gas flow, and O-ring connectors to accommodate end and side windows and the electrical feedthrough for the electret microphone/preamplifier (Xnowles, BT-1759). The output from the microphone/ preamplifier was further amplified (Tektronix mode $\mathrm{AM} 502$ ) and captured with a transient recorder (Biomation model 805) interfaced to the Nicolet signal averager. By positioning the microphone near the laser beam, the initial acoustic wave generated by the absorbed energy strikes the microphone before the waves reflected from the cell walls can cause interference; thus, only the first maximum in the complex signal was deemed significant. Experiments were repeated (16-256 shots) until a statistical precision of $\pm 2 \%-3 \%$ was achieved.

The optoacoustic method was also adapted for measurement of yield vs fluence data at pressures too high for the VLP $\Phi$ technique. The procedure was to introduce a known TFE/argon mixture into the optoacoustic cell and measure the OA signal at a low fluence. The gas mixture was then irradiated for a known number of laser shots at the higher fluence to be investigated; the static gas mixture was depleted by this irradiation. Following irradiation, the OA signa was again measured at low fluence to determine the amount of depletion, from which the IRMPD yield-per-shot was determined, the reaction products (at room temperature) do not interfere by absorption of laser light. Small air leaks limited the durations of data acquisition runs and the lowest pressures attainable, but useful data were obtained, as described in the Results section.

1,1,2-trifluoroethane was obtained commercially (PCR, Inc.) and was used after degassing in a grease-free high vacuum line. Gas purity was checked with a gas chromatograph/ mass spectrometer (Varian, Inc.) and the major impurity was found to be 1,1,1-trifluoroethane (about $0.1 \%$ ).

\section{RESULTS}

\section{IRMPD yields vs fluence}

The two versions of the VLP $\Phi$ technique were used to obtain most of the data on IRMPD yields, but these methods were complemented by the optoacoustic technique, which is suitable for measuring the effect of pressure. High fluence results were obtained using the Lumonics laser and lower fluence measurements were made with the high repetition rate Tachisto laser. At high fluences, the principal source of error was the fluctuation of laser power; long-term drift and instability of the mass spectrometer were the limiting factors at low fluence. The minimum uncertainty achieved with the single-shot accumulation method corresponds to about $\pm 2 \%-3 \%$, as determined in blank runs. Yields of that order, or lower were not reliably measured using the singleshot accumulation method in our experiments. However, by using the high repetition rate laser, each parcel of gas entering the VLP$\Phi$ cell is irradiated 10-12 times during its residence time, improving the precision of the yield measurements by a factor of 5-10.

The results obtained at $1079.85 \mathrm{~cm}^{-1}$ using both methods and both lasers show very good consistency, even when the laser beam mode structure is deliberately varied. As a test, the area of irradiation was changed from 2.7 to 0.78 $\mathrm{cm}^{2}$, using the Lumonics laser, and the results were indistinguishable from those obtained using a $0.42 \mathrm{~cm}^{2}$ beam from the Tachisto. Because of the insensitivity of the results to beam spatial modes, deconvolution ${ }^{12}$ of the spatial profile was not attempted, although an iterative approach based on the calculated results would be feasible (and time consuming).

Tests were carried out to eliminate other possible sources of experimental artifacts. For example, the residence time in the cell was held constant at $0.88 \mathrm{~s}$ and the cell pressure was varied from 0.8 to $20 \mathrm{~m}$ Torr without significantly affecting the results obtained; at higher pressures, the yields decreased slightly. In another test, the gas flow rate was held constant and the residence time was varied from 0.10 to 0.56 $\mathrm{s}$, without affecting the results.

All of these data are presented in Fig. 1, where the data sets show excellent consistency. The data are plotted on probability graph paper as a log-normal cumulative distribution, because it has been shown that for many IRMPD theoretical models and some experimental data, such a presentation gives straight lines. ${ }^{14}$

The optoacoustic measurements of yield vs fluence were carried out at pressures ranging from $1.5 \mathrm{~m}$ Torr TFE diluted in $33 \mathrm{~m}$ Torr argon, up to pressures as high as 10 Torr, as presented in Fig. 2. The most extensive optoacoustic data set corresponds to a total pressure of about $35 \mathrm{~m}$ Torr. These data show the same fluence dependence as the collision-free data, except that the absolute yields are lower, due to the

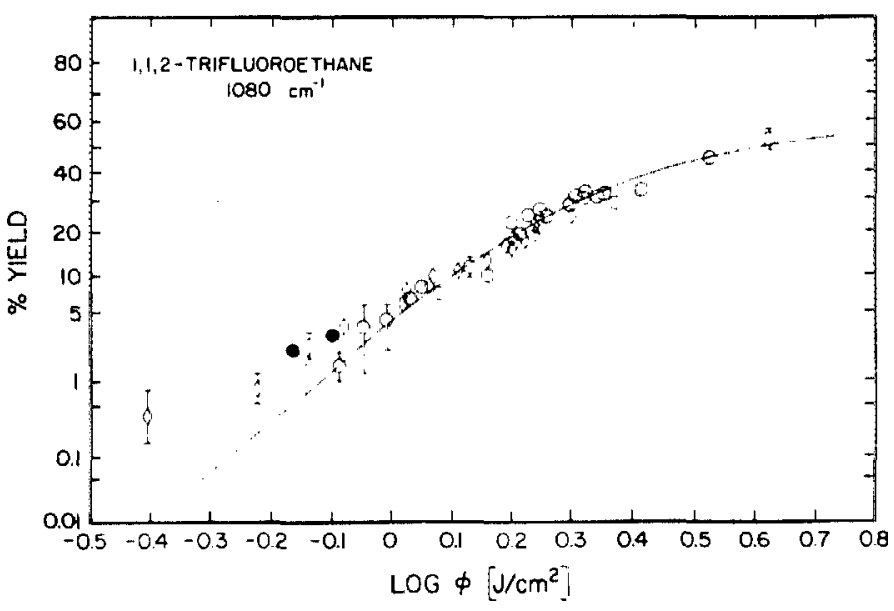

FIG. 1. Decomposition yields vs fluence at $1079.85 \mathrm{~cm}^{-1}$. Key: $\mathrm{O}-\mathrm{Lu}-$ monics laser; $\diamond-$ Tachisto laser; - Tachisto laser, very large number of laser shots. 


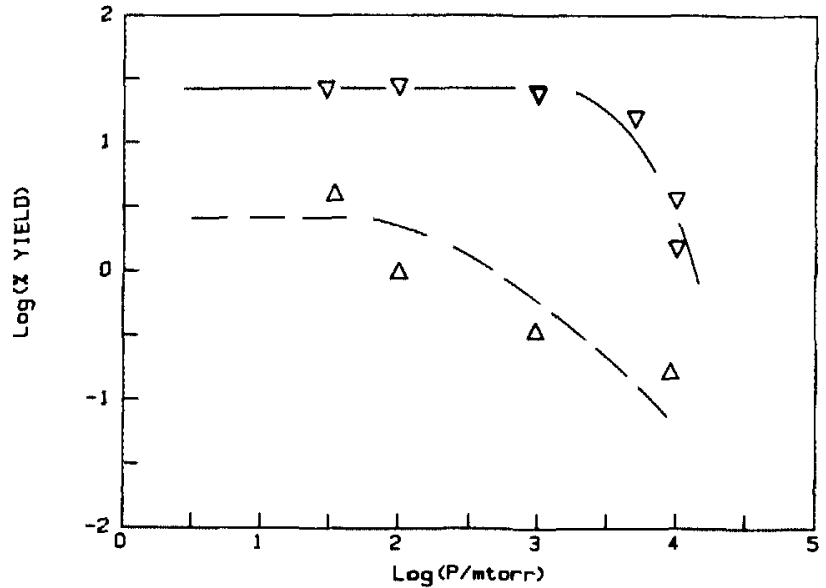

FIG. 2. Decomposition yield dependence on pressure, determined by the optoacoustic method. The curves are schematic. Upper curve: $2.7 \mathrm{~J} \mathrm{~cm}^{-2}$; absolute uncertainties are $\pm 2 \%$. Lower curve: $0.9 \mathrm{~J} \mathrm{~cm}^{-2}$; absolute uncertainties are $\pm 0.2 \%$.

higher pressures used and, perhaps, to small systematic errors in the fluence measurements.

The two series of optoacoustic measurements presented in Fig. 2 show the different effects of pressure on IRMPD yields at different laser fluences. At high fluence, the yield is not greatly affected, except at relatively high argon pressures. At low fluences, however, the yields are strongly affected by argon pressure over the entire range of pressure investigated.

\section{Optoacoustic measurements of absorbed laser power Calibration}

The optoacoustic signals depend on gas composition, pressure, collisional energy transfer rates, laser energy, beam diameter, and spatial position of the microphone [see Ref. 5(d) for a fuller discussion], and careful calibration was necessary. Absolute calibration factors were obtained by using the absorption cross section as measured over a path length of $30.5 \mathrm{~cm}$ and using the Tachisto laser and the Scientech power meter; Beer's Law fully described the experimental data. The absorption cross section measured in this way $\left[(3.46 \pm 0.35) \times 10^{-9} \mathrm{~cm}^{2}\right]$ is about a factor of 2 larger than that measured with a Digilab FTIR with $0.1 \mathrm{~cm}^{-1}$ resolution $\left[(2.0 \pm 0.2) \times 10^{-19} \mathrm{~cm}^{2}\right]$. We concluded that the laser measurement is more pertinent to our experiments, considering the complex spectrum (Fig. 3) and possible coupling mechanisms that may be operative at high laser power.

At sufficiently low fiuence, most molecules absorb no more than one photon and decomposition is not important. Extensive calibrations were carried out at low fuences by measuring the microphone signal for a fixed set of "hardware parameters" (e.g., fluence, laser beam diameter, and location of the microphone) and a fixed partial pressure of $T F E$ diluted in various pressures of argon. The parameters were varied systematically to determine the saturation limit of the microphone and the effects of the parameters. For fixed laser beam spatial parameters and for TFE highly diluted in argon, the microphone signal was found to depend primarily on absorbed laser energy and slightly on argon pressure. The instrument response was directly proportional to absorbed laser power as long as the microphone was not overloaded. The calibration factors were found to depend linearly on $\log$ (pressure) for a wide range of experimental parameters, as shown in Fig. 4, where the calibration factor is given by ${ }^{5(d)}$

$$
F=S_{\mathrm{OA}} C_{M} / E_{A} \text {. }
$$

$\mathrm{H}_{\mathrm{H}}$ ere, $S_{\mathrm{OA}}$ is the microphone signal (arbitrary voltage units), $C_{M}$ is the total heat capacity of the gas mixture at constant volume, and $E_{A}$ is the absorbed energy per unit length. Based on Beer's law at the small absorbance limit and the measured cross section $\sigma, E_{A}$ is given by

$$
E_{A}=E_{L} \sigma[\mathrm{TFE}] \text {, }
$$

where $E_{L}$ is the laser pulse energy and [TFE] is the TFE concentration in convenient units. For interpretation of the absorbed laser energy measurements, a least-squares fit to

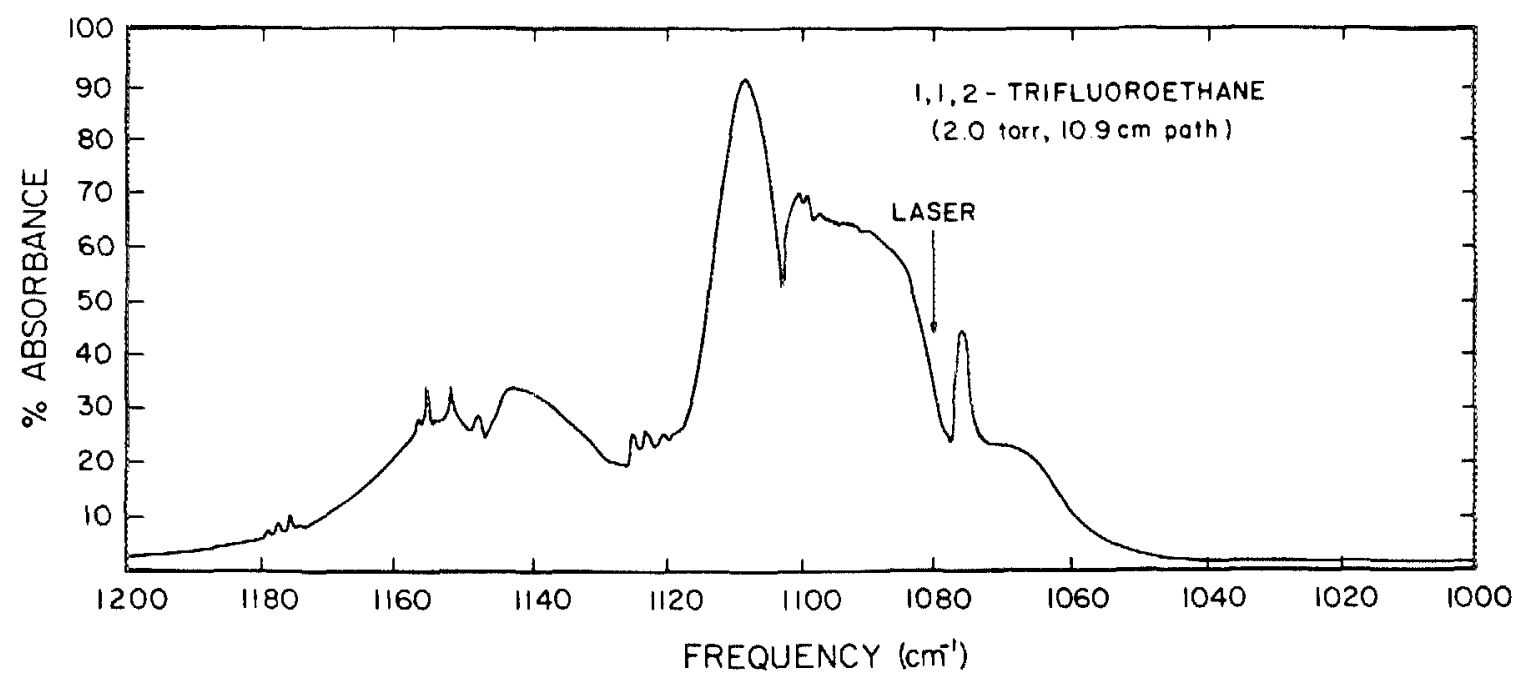

FIG. 3. Infrared spectrum of 1,1,2-trifluoroethane near $1100 \mathrm{~cm}^{-1}$. FTIR spectrum obtained with $\approx 0.1 \mathrm{~cm}^{-1}$ resolution; for vibrational assignment, see Ref. 8. 


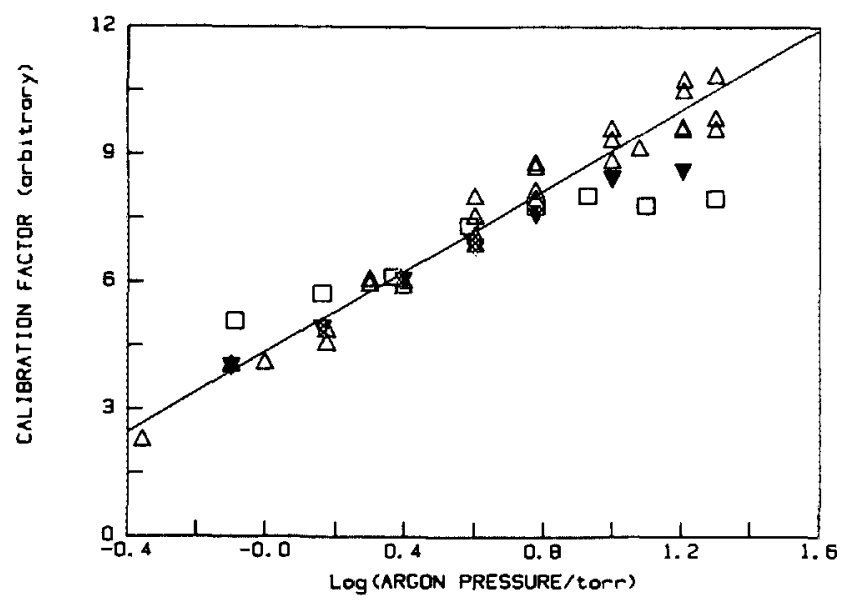

FIG. 4. Optoacoustic signal calibration curves. Pressures of TFE: $\triangle-2.4$ $24 \mathrm{mTorr} ; \boldsymbol{\nabla}-50 \mathrm{mT}$ Trr; $\square-125 \mathrm{mT}$ Torr; solid line: least-squares fit to low-pressure points.

the experimental calibration data was used, as shown in the figure.

\section{Absorbed laser energy measurements}

Using the experimental calibration curve, we can determine the absorbed energy, based on the observed microphone signal and the gas composition. To place the absorbed energy on the molecular scale, it is conveniently expressed as the average number of absorbed photons per molecule, given by

$$
\langle n\rangle=S_{\mathrm{OA}} C_{M} /\left(F h v_{0}[\mathrm{TFE}]\right),
$$

where $h v_{0}$ is the laser photon energy and the other factors have been identified above. Using this expression places the absorbed energy on a reduced scale, enabling direct comparisons of absorbed energies obtained under widely different conditions.

Experiments were carried out to determine the effects of added argon and laser fluence on $\langle n\rangle$. Usually, better results were obtained in static experiments than in flowing gas systems, which suffered from fluctuations in total pressure and in gas composition. Care was taken to avoid depletion of the TFE in experiments with high laser fluence. The results are presented in Fig. 5, and they show no significant dependence on pressure for fluences up to about $2 \mathrm{~J} \mathrm{~cm}^{-2}$. The results are highly consistent, with a relative uncertainty of about $\pm 5 \%$ and an absolute uncertainty estimated at about $\pm 30 \%$, mostly due to the uncertainty in the fluence and energy measurements that went into calibration of the microphone.

At the highest fluences, decomposition of the TFE during the laser pulse may become significant, affecting the simple interpretation of the results. Although the reactions are slightly endothermic and can affect the optoacoustic signal to a small extent, the major uncertainty is due to the unknown extent of absorption of the laser photons by vibrationally hot reaction products. In the infrared fluorescence measurements on this system, ${ }^{7}$ direct evidence was obtained for secondary photolysis of the products.

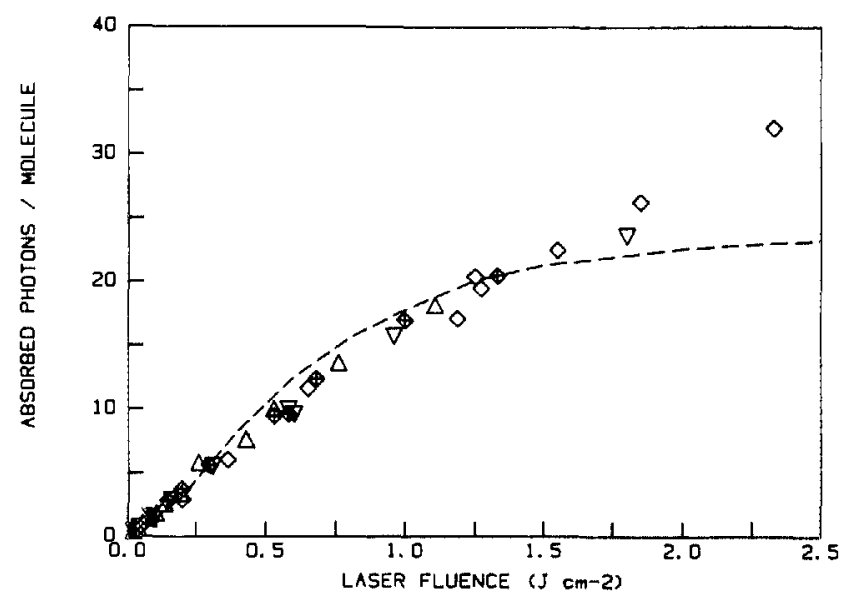

FIG. 5. Average number of photons absorbed per molecule vs fluence. Argon pressures: $\nabla-0.5$ Torr; $\diamond-1.0$ Torr; $\triangle-20.0$ Torr; $\diamond-10.0$ Torr; dashed line: Master Equation calculation.

\section{MASTER EQUATION MODEL}

The basic Master Equation approach to simulating experiments such as these has been described in detail, ${ }^{6}$ except for several significant improvements. Essentially, the approach ${ }^{15}$ used Monte Carlo techniques to simulate random walks of individual molecules in energy space, subject to the properties of the molecule and its interactions with the laser photons. ${ }^{16}$ Many such "trajectories" are calculated and the results are averaged, or otherwise stored for examination. Other than that used in calculating the vibrational densities of states, no intrinsic "graining" is imposed on the physics of the system, unlike approaches that use an "energy-grained" Master Equation. Thus, convergence of the solutions does not depend on grain size, although the precision obtainable depends on the number of trajectories calculated, as is usual in Monte Carlo simulations. ${ }^{16}$

The physical processes consicered in the model include optical absorption and stimulated emission, collisional energy transfer, infrared fluorescence, and up to three unimolecular reaction channels; several models for some of these processes have beer incorporated in the computer code as options. ${ }^{6}$

The Master Equation implementation has been significantly improved in several ways for present purposes. First, all densities of vibrational states are now calculated from exact counts of states, ${ }^{17}$ rather than from the Whitten- $\mathbb{R a}$ binovitch approximation ${ }^{18}$ used earlier. For TFE, an energy grain of $25 \mathrm{~cm}^{-1}$ has proved to be adequate for the calculations. The densities of states for the first $2500 \mathrm{~cm}^{-1}$ of internal energy are stored in a 100-element array; in addition, the densities for the first $50000 \mathrm{~cm}^{-1}$ are stored in a second 100 element array. The censity of states at any energy up to $50000 \mathrm{~cm}^{-1}$ is selected by interpolation between elements in one of these two arrays. Incorporation of exact state counts has made little difference in computed results, except for the distribution of initial energies selected. For example, now the thermal distribution is much more accurately produced, compared to the somewhat shifted version obtained ${ }^{6}$ using the Whitten-Rabinovitch approximation. 
The other improvements are concerned with the optical pumping and stimulated emission segments of the model. In addition to the former capability to consider a laser intensity that is constant for the entire duration of a trajectory and a laser pulse shape that decays exponentially, additional provision has been made for a laser pulse of constant duration and finite length. This capability enables investigation of post-pulse phenomena subsequent to a pulse of constant intensity. The second enhancement of the optical interaction model was to incorporate Quack's theory of infrared laser absorption/emission for his cases B and C. ${ }^{19}$

Prior to incorporation of Quack's theory in our model, we used a semiempirical expression for the absorption cross section dependence on vibrational energy. $4,6,14,16$

$$
\sigma(E)=\sigma_{0}\left[1+E / h v_{0}\right]^{n(\lambda)},
$$

where $\sigma_{0}$ is the absorption cross section for all molecules at the zero-point energy level, $h v_{0}$ is the laser photon energy, and $n(\lambda)$ is a parameter that depends on the wavelength of the laser. This expression has been used in Master Equation treatments of IRMPD with fair success, but recently, Haas and co-workers commented on the inadequacies of a Master Equation treatment that employed this type of expression. ${ }^{2}$ In order to reduce the degree of empiricism somewhat, and because we also find Eq. (5) to be inadequate for treatment of the results obtained in the present work (see below), we implemented the Quack formalism for cases B and C.

Quack has described his statistical-dynamical theory extensively in the literature ${ }^{19}$ and we will not attempt to repeat the details here. His approach is to evaluate the matrix elements for laser pumping and stimulated emission using a combination of $a b$ initio theory and judicious approximations. The resulting expressions give the optical transition rates in terms of physically measurable quantities, with provision for empirical parametrization, where desirable.

According to Quack, ${ }^{19}$ the optical transition rates for up-pumping from level $M$ to $M+1$ for both cases $\mathrm{B}$ and $\mathrm{C}$ are given by the expression

$$
K_{M+1, M}=2 \pi\left|V_{M+1, M}^{0}\right|^{2} / \delta_{M+1},
$$

where $\delta_{M+1, M}$ is the frequency spacing of effectively coupled states in the upper level and $\left|V_{M+1, M}^{0}\right|$ is the absolute value of the matrix element connecting the two levels (each microcanonical level contains many individual quantum states). According to Quack, $\delta_{i}$ can be related to the total density of vibrational states according to the expression

$$
\delta_{i}=2 \pi c / \rho\left(E_{i}\right) \text {, }
$$

where $c$ is the speed of light, and $\rho\left(E_{i}\right)$ is the total density of vibrational states at the energy of levell $i$. The matrix element is related to the laser intensity $I$ and the integrated absorption cross section $\left\{\int \sigma d x\right\}$, by the expression

$$
\left|V_{M+1, M}^{0}\right|^{2}=\alpha \xi\left(E_{M}\right) \frac{\rho^{\prime}\left(E_{M}\right) I\left\{\int \sigma d x\right\}}{\rho\left(E_{M+1}\right) \rho\left(E_{M}\right) \Delta E_{Q} h v_{p}},
$$

where $\rho^{\prime}(E)$ is the density of vibrational states with the pumped vibrational mode in the zero level, $\Delta E_{Q}$ is the level width over which the matrix elements are approximately constant, and $h v_{p}$ is the energy (expressed in $\mathrm{cm}^{-1}$ ) of the pumped mode. The factor $\xi\left(E_{M}\right)$ allows empirical parame- trization of the expression; when $\xi\left(E_{M}\right)=1.0$, the parameters are assumed to be independent of internal energy and to equal those measured at low intensities. For intensities expressed in $\mathrm{W} \mathrm{cm} \mathrm{cm}^{-2}$, state densities in states per $\mathrm{cm}^{-1}, \sigma$ in $\mathrm{cm}^{2}, \Delta E_{Q}$ in $\mathrm{cm}^{-1}$, and $d x$ in $\mathrm{cm}^{-1}$, the proportionality constant is $\alpha=1.509 \times 10^{33}$, to express the squared matrix element in units of $\mathrm{rad}^{2} \mathrm{~s}^{-2}$.

The down-pumping rates differ for cases $B$ and $C$. For case $B$, the down-transition rate from level $M$ to $M-1$ is given by

$$
K_{M-1, M}^{\mathrm{B}}=K_{M-1, M} \frac{\rho\left(E_{M-1}\right)}{\rho\left(E_{M}\right)},
$$

where the factors have been defined above. For case $C$, the down-transition rate is given by

$$
\begin{aligned}
K_{M-1, M}^{\mathrm{C}} & =K_{M-1, M}^{\mathrm{B}} \frac{2 \delta_{M}}{\left(\pi V 3\left|V_{M, M-1}^{0}\right|^{2}\right)} \\
& =\gamma K_{M-1, M}^{\mathrm{B}}\left[\frac{\rho\left(E_{M-1}\right) \Delta E_{Q} h v_{p}}{\rho\left(E_{M}\right) \rho^{\prime}\left(E_{M-1}\right) I\left\{\int \sigma d x\right\}}\right]^{1 / 2},
\end{aligned}
$$

where the symbols have the same meanings and units as above, and the proportionality constant $\gamma$ is $1.784 \times 10^{-6}$ for the transition rate expressed in $\mathrm{s}^{-1}$. The crossover from case $\mathrm{C}$ to case $\mathrm{B}$ at low energy and/or low intensity is treated by selecting the larger of the two calculated transition rates, as described by Quack.

For 1,1,2-triffuoroethane, the molecular parameters needed for use of the Quack expressions are known and are summarized in Tables I and II. The expressions can be empirically adjusted ${ }^{19}$ by treating $\Delta E_{Q}$ as a parameter used in concert with $\xi(E)$, as discussed below. Densities of vibrational states were obtained from exact counts by using the vibrational assignment for the molecule and assuming harmonic oscillators. For the density of states calculations, the internal rotor was treated as a $117 \mathrm{~cm}^{-1}$ vibration.

\section{DISCUSSION}

The two experimental techniques employed in this investigation provide information about the IRMPA process over two complementary internal energy ranges. The optoacoustic measurements are most accurate for low fluences, or when reaction is not important. On the other hand, the IRMPD measurements require that molecules be pumped above the reaction threshold, and it has been shown that the technique provides information mostly about the reaction threshold region and above. ${ }^{4,14,16}$

The absorbed energy measurements show little dependence on pressure, and thus we conclude that at low pressures, where collisional deactivation during the pumping process is unimportant, the measurements reflect the average energy of the nascent population. Since the reaction threshold energies are about $24000 \mathrm{~cm}^{-1}$, the optoacoustic measurements can be interpreted in a straightforward way up to $\langle n\rangle \approx 22$. Above that value, the extent of decomposition will affect the results in three ways: (1) depletion of the parent molecules during the laser pulse will mean fewer photons absorbed, (2) the reaction thermochemistry can affect 
TABLE I. Molecular properties of 1,1,2-trifluoroethane.

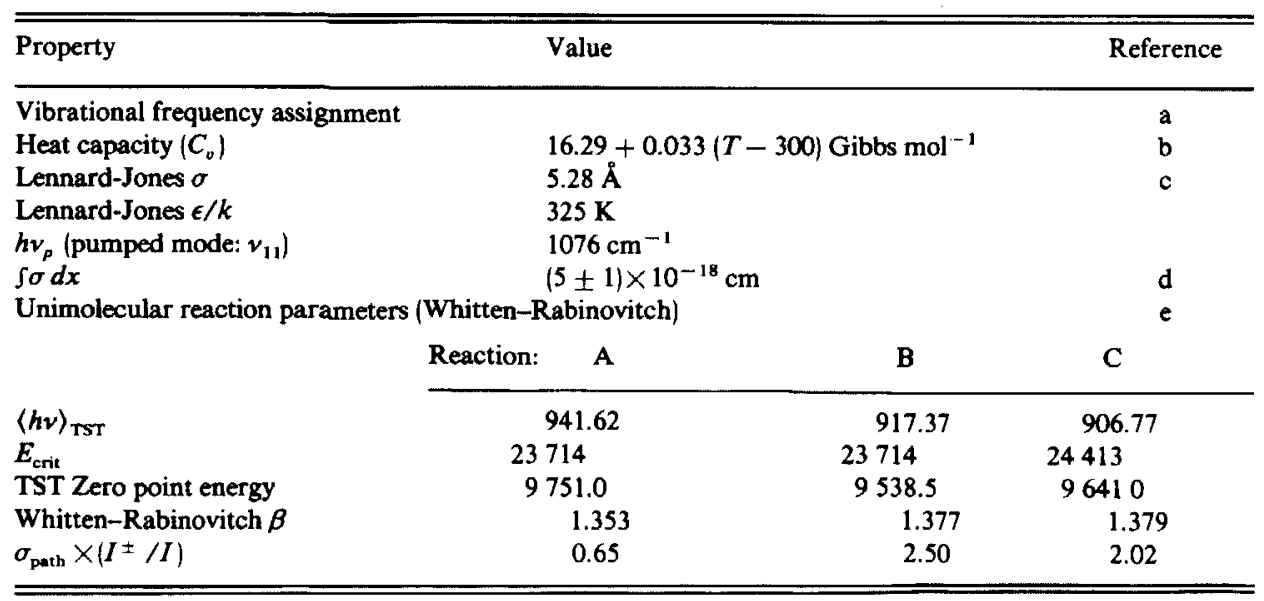

V. F. Kalasinsky, H. V. Anjaria, and T. S. Little, J. Phys. Chem. 86, 1351 (1982).

${ }^{b}$ Calculated from the vibrational assignment.

'Estimated using Lyderson's method as described in R. C. Reid and T. K. Sherwood, The Properties of Gases and Liquids, 2nd ed. (McGraw-Hill, New York, 1966).

${ }^{d}$ Measured in this work; see FTIR spectrum in Fig. 3.

"Whitten-Rabinovitch parameters for the transition states were derived from the detailed transition state frequencies given by B. E. Holmes, D. W. Setser, and G. O. Pritchard, Int. J. Chem. Kinet. 8, 215 (1976); RRKM $k(E)$ 's were calculated using the TFE state densities and Whitten-Rabinovitch approximation for the sums of states.

the heat release, and (3) the [hot] reaction products produced during the pulse, or in previous pulses, may absorb the laser light. Inspection of Fig. 5 shows that with 10 Torr argon added, $\langle n\rangle \geqslant 32$, much higher than the reaction threshold energy. At this high pressure, decomposition is inhibited by collisional deactivation of excited molecules and the reactant is not depleted. Indeed, the collisional deactivation rate is fast enough so that the excited molecules are never pumped very high on the energy ladder.

The absence of a pressure dependence at relatively high pressures, where collisional deactivation during the laser pulse is important, indicates that the net optical absorption rate of the excited molecules does not depend strongly on internal energy. Inspection of the 10 Torr added argon data set in Fig. 5 shows that it is virtually indistinguishable from data sets obtained at much lower pressures. More quantitative conclusions about the optical pumping process require the numerical calculations described below.

The IRMPD measurements probe molecules pumped above the reaction threshold. When the yields are low, the decomposing molecules represent only the high energy "tail" of the population distribution, which is highly sensitive to several effects, including the optical pumping process and collisional energy transfer. At sufficiently low pressures, homogeneous collisional effects are unimportant, and the IRMPD yields reflect the fraction of molecules pumped above the reaction threshold that react before deactivation at

TABLE II. Quack theory fitted parameters.

\begin{tabular}{cc}
\hline \hline Parameter & Value \\
\hline Pulse width & $125 \pm 15 \mathrm{~ns}$ \\
$\Delta E_{Q}$ & $4 \pm 1 \mathrm{~cm}^{-1}$ \\
$W$ & $23700 \pm 100 \mathrm{~cm}^{-1}$ \\
$m$ & $20 \pm 5$ \\
\hline
\end{tabular}

the walls of the cell. Since drift times to the cell walls are of the order of $3 \times 10^{-4} \mathrm{~s}$, the decomposition yield at low pressure equals the fraction of molecules that attain energies high enough to have specific unimolecular rate constants $k(E)>3 \times 10^{3} \mathrm{~s}^{-1}$ (i.e., $E \geqslant 26000 \mathrm{~cm}^{-1}$ ).

Information about the optical pumping process can be obtained from the dependence of the collision-free IRMPD yield on fluence. As a class, systems with optical pumping properties that can be described by the simple power-law equation (5) have decomposition yields that can be plotted linearly vs $\log$ (fluence) on probability graph paper, as has been discussed elsewhere. ${ }^{14,16}$ The VLP $\Phi$ experimental data are presented on such a log-normal plot in Fig. 1 and the resulting line is clearly curved, indicating that the optical pumping is not well described by Eq. (5). The heuristic approach taken in our earlier work in order to account for such curvature was to assume that the excited molecules were partitioned into two or more population subsets with different optical pumping parameters. ${ }^{20}$ This approach could have been taken in the present work, but Quack's more sophisticated theory ${ }^{19}$ accounts satisfactorily for the experimental observations without recourse to the multiple-population model.

A more complete discussion of collisional energy transfer in this system will appear elsewhere, ${ }^{21}$ but IRMPD yield data obtained with the optoacoustic technique are presented in Fig. 2. These results show that, as expected, the low fluence data are far more susceptible to collisional effects than data obtained at higher fluences. The high energy tail of the excited molecule distribution is sensitively affected by collisions, even at pressures as low as 35 mTorr. (Note that our experimental tests showed that the VLP $\Phi$ data obtained at $20 \mathrm{~m}$ Torr were not significantly affected by total pressure.) The decreased yield is due almost entirely to "postpulse" collisional deactivation of excited molecules that have energies only a little above the reaction threshold. In 
addition, mass transport to the cell wall is affected by the higher pressures, and the detailed differences in the collision efficiency of walls vs gas phase must be taken into account for a full description. ${ }^{22}$ Such a description is feasible, but it is beyond the scope of the present work.

To obtain more quantitative information about the pumping process, the Master Equation approach described above was utilized and the effects of many parameters were investigated. Insofar as possible, experimentally measured parameters were used in order to limit the latitude of the calculations. Many combinations of assumptions and input parameters were investigated to determine whether they were consistent with the experimental data, but it was found that the two sets of experimental data combined with knowledge of TFE molecular properties constrained the latitude of the calculations greatty, and a satisfactory simulation of the experiments was difficult to achieve.

The unsatisfactory attempts at simulation of the experiments included the following combinations of assumptions:

(1) Exponential or square laser pulse used with the power-law absorption cross section in Eq. (5): This combination could be made to fit the absorbed energy measurements very well by using the measured cross section and adjusting the parameter $n(\lambda)$, but the predicted yield vs fluence results were too steep on a log-normal plot, as shown in our preliminary report. ${ }^{23}$ Also, the simulated log-normal plot was a straight line, unlike the experimental data.

(2) Exponential laser pulse and Quack's theory with $\xi(E)=1.0$ : Here, the absorbed energy measurements could be well simulated by varying the parameter $\Delta E_{Q}$, but the predicted reaction yields were much too high. Furthermore, the pulse decay time significantly affected the predicted value of $\langle n\rangle$ at a given fluence. This is because the transition between cases $B$ and $C$ depends on laser intensity, and the down-pumping rate in case $C$ is much greater than in case $B$. Molecules pumped up, early in the pulse under case $B$ conditions, could be rapidly down pumped later in the pulse, when the intensity is lower and case $C$ is operative. This effect may be observable in carefully designed experiments; its presence may indicate that an accurate simulation of the laser pulse is necessary for more detailed calculations.

(3) Square laser pulse and Quack's theory with $\xi(E)=1.0$ : To simplify the model, a square pulse was assumed. This simplified version gave good agreement with $\langle n\rangle$ for suitable choices of $\Delta E_{Q}$ and the pulse width, but the predicted reaction yields were much too high, indicating that the average molecules could be well modeled, but the high-energy tail of the distribution (that resulted in decomposition) was predicted to be too highly populated.

Since the Quack theory with $\xi(E)=1.0$ (and appropriate choices of $\Delta E_{Q}$ and laser pulse width) fitted the experimental data on absorbed laser energy very well, our approach was to choose a functional form that gave $\xi(E)=1.0$ at low internal energies, but allowed simple variations at higher energies. A first attempt at a functional form for $\xi(E)$ incorporated a Lorentzian with "width" and "center" that could vary linearly as a function of internal energy, as suggested by Quack. ${ }^{\text {19(d) }}$ This approach was not successful, although nonlinear dependencies on internal energy could probably be made to work. Inspection of the infrared spectrum of TFE (Fig. 3) shows that at least two fundamental transitions are important, and a simple Lorentzian is not adequate.

The second attempt at choosing a functional form for $\xi(E)$ was successful. The assumed form incorporates only two empirical parameters and it gives $\xi(E)=1.0$ at low energies:

$$
\xi(E)=\exp \left\{-(E / W)^{m}\right\}
$$

Although this function is arbitrary and not unique, it provides a smooth transition between the optical properties of the molecule at low energies, where they can be measured with a spectrometer, and at high energies, where the anharmonicities may become very significant.

By a process of trial and error, a combination of parameters (Table II) was found that gives model simulations in good agreement with the experimental data sets, as shown in Figs. $l$ and 5 . The calculated yield vs fluence results are in excellent agreement with the experimental data over virtually the entire fluence range investigated. The minor discrepancy at very low fluence may be due to experimental artifacts and/or to inadequacies of the model for the extremely small populations at the highest part of the distribution. The calcu-

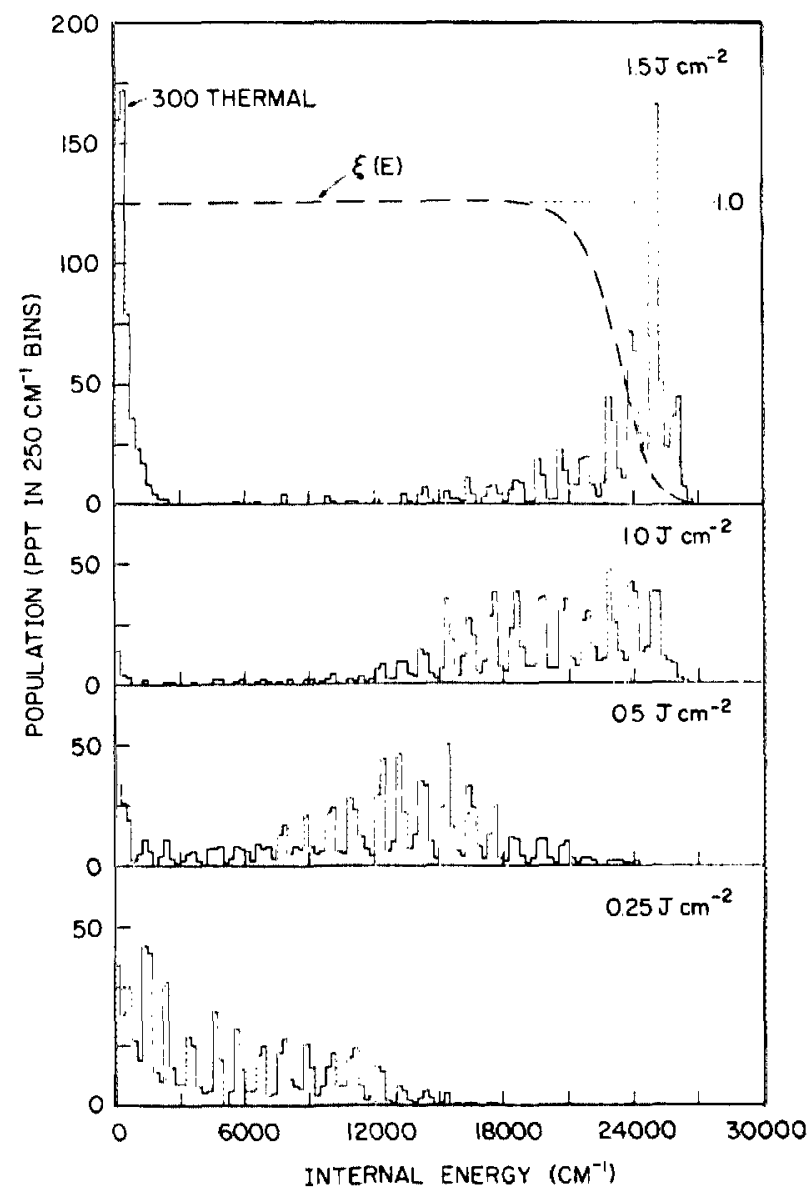

FIG. 6. Population distributions. Each panel corresponds to a different laser fluence. For reference, the $300 \mathrm{~K}$ thermal distribution and the function $\xi(E)$ are shown in the top panel (the population distribution for $1.5 \mathrm{~J} \mathrm{~cm}^{-2}$ is essentially zero below $5000 \mathrm{~cm}^{-1}$ ). 
lated curve for $\langle n\rangle$ vs fluence in Fig. 5 corresponds to an assumed argon pressure of $1.0 \mathrm{Torr}$, and the saturation effect due to the function $\xi(E)$ is clearly seen; at higher pressures, molecules are deactivated before reaching such high energies, and the predicted behavior then resembles that observed in the experiments with 10 Torr argon.

The fitted parameters appear to be reasonable, but they are not unique, because they depend on the assumed functional form for $\xi(E)$. Within the framework of our assumptions, however, there is very little latitude for variation of the parameters, as indicated by the estimated uncertainties.

Comparison of the parameters in Table II with the known molecular properties of TFE and the experimental conditions shows good agreement. For example, the laser pulse width of $125 \pm 15$ ns for a simulated square pulse is roughly similar to the typical $\mathrm{CO}_{2} \mathrm{TEA}$ laser pulse, which is comprised of many irratically modulated spikes enclosed by an average amplitude envelope consisting of a $100 \mathrm{~ns}$ pulse with a $1-2 \mu \mathrm{s}$ tail. Also, the parameter $\Delta E_{Q}$ is expected to be of the same order as the width of the infrared absorption band ${ }^{19}$; in TFE, the laser photons are $4 \mathrm{~cm}^{-1}$ out of resonance with the $v_{11} Q$ branch (for the $C_{1}$ conformer, the predominant form in the gas phase) at $1076 \mathrm{~cm}^{-1}$, which is about $2.5 \mathrm{~cm}^{-1}$ in width, compared to $\Delta E_{Q}=4 \pm 1 \mathrm{~cm}^{-1}$. Most interesting, however, is the fitted value of the parameter $W$.

In Eq. (12), the parameter $W$ is the energy at which $\xi(E)=1 / e$ and the optical coupling matrix element is much reduced compared to its value for transitions at low internal energies. There can be several reasons for a reduction in magnitude of the matrix elements, but a good explanation is provided if the transition energies shift away from resonance due to anharmonicity. The value of $W$ depends slightly on that of the parameter $m$, but for $m=20$, the uncertainty in $W$ is quite small.

The fact that the value of $W$ is so close to the reaction thresholds is highly suggestive that anharmonicity introduced by the open reaction channels plays an important role in the spectroscopic properties of this highly vibrationally excited molecule. Although $\xi(E)$ is not a unique function, its general behavior may provide a good semiquantitative description of the optical matrix elements near the reaction thresholds. Based upon our experience in attempting to simulate the experimental cata presented here, we conclude that, within the framework of the Quack theory, the matrix elements must undergo a drastic change near the reaction thresholds. If our conclusion is correct, this may be the first time the effect has been observed in highly vibrationally excited polyatomic molecules.

The reactions in TFE are HF eliminations that must pass through molecular configurations starting from singlebonded carbon atoms with $s p^{3}$ hybridization, through intermediate configurations (i.e., a transition state), to doubly bonded carbon atoms with $s p^{2}$ hybridization. The $v_{11}$ infrared transition at $1076 \mathrm{~cm}^{-1}$ is an asymmetric $\mathrm{C}-\mathrm{F}$ stretching mode, a mode that must be affected by a change in hybridization of the carbon atom: as the hybridization changes from $s p^{3}$ to $s p^{2}$, the C-F bond will be slightly strengthened, resulting in changes in vibrational frequen- cies. Specifically, the $1076 \mathrm{~cm}^{-1} \mathrm{C}-\mathrm{F}$ stretch mode frequency in TFE may be compared to the C-F stretch modes in cisand trans-1,2,-diffuoroethylene (product molecules), which have frequencies of $1014,1127,1123$, and $1159 \mathrm{~cm}^{-1}$, far out of resonance with the laser photons. Since every $\mathrm{C}-\mathrm{F}$ bond in TFE (even those not directly involved in the HF elimination) undergoes a change in hybridization when reaction occurs, it is not surprising that the transition matrix elements for the C-F stretch modes drastically decrease in magnitude at energies close to the reaction thresholds.

The primary objective of this work was to determine the population distributions produced in TFE by IRMPA. A1though not unique, the model results probably represent a very good approximation to the distribution functions, especially considering the narrow latitudes available for satisfactory simulation of the two data sets. The population distributions predicted for several laser fluences are presented in Fig. 6 along with a plot of $\xi(E)$. Note that the $250 \mathrm{~cm}^{-1}$ "binning" of the calculated results was for bookkeeping purposes and had no effect on the actual calculation.

The calculated distributions predict that at low and intermediate fluences, a significant fraction of molecules remains in the initial thermal distribution, but that fraction becomes much smaller at higher fluences. At all fluences, the initial thermal distribution is translated upwards by integral numbers of photon energies, resulting in a series of peaks that have about the same width as the initial thermal distribution. Also, the overall dispersion of energies is quite large, compared to the mean. At high fluences, however, the dispersion becomes much smaller, as molecules "stack up" at high energies, due to the fall off of $\xi(E)$. On a longer time scale, most molecules will decompose, because they have energies greater than the reaction thresholds.

These population distributions illustrate that IRMPA is effective in exciting most molecules in the laser beam and that the distributions are quite broad. For effective use of the technique in laboratory studies of other molecules, efforts are necessary to elucidate the distribution functions produced. Absorbed energy measurements taken alone are not sufficient to determine the population distributions, since any of the combinations of assumptions described above could simulate the optoacoustic experiments.

The combinations of experimental measurements that are most effective in constraining the computer simulations are those that measure different attributes of the population distributions. For example, in the present study, the absorbed energy measurements gave information mostly about the mean energy (the first moment of the population distribution), while the decomposition yield vs fluence measurements probed the high energy tail of the distribution function. In Fig. 7, $\langle E\rangle$ is plotted as a function of $\langle n\rangle$ to illustrate the direct connection between the first moment of the population distribution and the experimental observable. Thus, the experimental approach can be based on measuring properties that depend on the individual moments of the distribution function sought.

Some experimental measures that have not been considered here are the chemical reaction branching ratios to form the isomeric ethylene products. Branching ratio measure- 


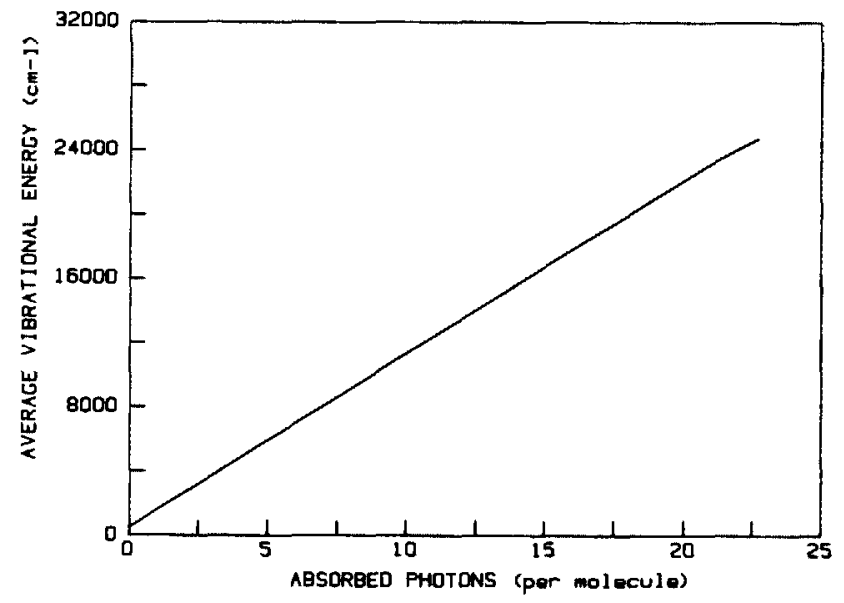

FIG. 7. Calculated values for $\langle E\rangle$ as a function of $\langle n\rangle$. Note that the average thermal vibrational energy of TFE at $300 \mathrm{~K}$ is about $470 \mathrm{~cm}^{-1}$.

ments are quite feasible in many systems and, in many cases, can give useful information. In this system, however, the reaction threshold energies are so close together that the relative yields are insensitive to small variations of the parameters in Table U. For example, variation of the parameter " $m$ " from $m=15$ to $m=25$ is calculated to change the relative yield of 1,1 -difluoroethylene from $\approx 0.14$ to $\approx 0.16$. This variation is too small to be useful in refining the parameter values in the present investigation.

In the present study, the strategy of using a combination of experimental techniques to measure different attributes has resulted in information about the population distributions formed by IRMPA. This information will be used in subsequent studies of collisional energy transfer ${ }^{21}$ and bimolecular reactions. In the following paper, ${ }^{7}$ the calculated population distributions are used to predict infrared emission intensities, which are then compared to those observed in experiments.

\section{ACKNOWLEDGMENTS}

The authors gratefully acknowledge financial support from the U.S. Army Research Office. JMZ acknowledges a fellowship from the Swiss Fonds National, and TCB thanks K. D. King at the University of Adelaide for his support and encouragement. Many of the numerical calculations were carried out in the Chemical Physics Laboratory at SRI using the VAX computing facility, which was provided by a grant from the National Science Foundation. The authors are happy to acknowledge the helpful conversations with $\mathfrak{D}$. W.
Setser, who also provided useful technical information on several molecules studied in his laboratory.

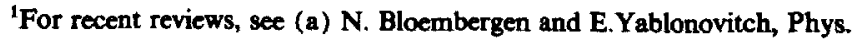
Today 31, 32 (1978); (b) P. A. Schultz, Aa. S. Sudbo, D. J. Krajnovich, H. S. Kwok, Y. R. Shen, and Y. T. Lee, Annu. Rev. Phys. Chem. 30, 379 (1979); (c) M. F. Goodman, J. Stone, and E. Thiele, in Multiple-Photon Excitation and Dissaciation of Polyatomic Molecules, edited by C. D. Cantrell (Springer, Berlin, 1980); (d) J. L. Lyman, G. P. Quigley, and O. P. Judd ibid:; (e) D. S. King, Dynamics of the Excited State, edited by K. P. Lawley (Wiley, New York, 1982), p. 105.

${ }^{2}$ S. Ruhman, O. Anner, and Y. Haas, J. Phys. Chem. 88, 6397 (1984).

${ }^{3}$ For example, see J. W. Hudgens and J. D. McDonald, J. Chem. Phys. 76, 173 (1982).

(a) D. M. Golden, M. J. Rossi, A. C. Baldwin, and J. R. Barker, Acc. Chem. Res. 14, 56 (1981); (b) M. J. Rossi, J. R. Barker, and D. M. Golden, J. Chem. Phys. 76, 406 (1982).

s(a) R. D. Bates, Jr., G. W. Flynn, and J. K. Knudtson, J. Chem. Phys. 53, 3621 (1970); (b) Opto-Acoustic Spectroscopy and Detection, edited by Y. H. Pao (Academic, New York, 1977); (c) N. Presser, J. R. Barker, and R. J. Gordon, J. Chem. Phys. 78, 2163 (1983); (d) J. R. Barker, L. Brouwer, R. Patrick, M. Rossi, P. L. Trevor, and D. M. Golden, Int. J. Chem. Kinet. (in press).

${ }^{6}$ (a) J. R. Barker, Chem. Phys. 77, 301 (1983); (b) J. R. Barker and R. E. Golden, J. Phys. Chem. 88, 1012 (1984).

'J.-M. Zellweger, T. C. Brown, and J. R. Barker, J. Chem. Phys. 83, 6261 (1985).

BV. F. Kalasinsky, H. Anjaria, and T. S. Little, J. Phys. Chem. 86, 1351 (1982).

'B. E. Holmes, D. W. Setser, and G. O. Pritchard, Int. J. Chem. Kinet. 8, 215 (1976), and references therein.

${ }^{10}$ D. M. Golden, G. N. Spokes, and S. W. Benson, Angew. Chem., Int. Ed. Eng. 12, 534 (1973).

"J. S. Chang, J. R. Barker, J. E. Davenport, and D. M. Golden, Chem. Phys. Lett. 60, 385 (1979).

${ }^{12}$ For a discussion of intensity profiles and deconvolution considerations, $\sec$ A. C. Baldwin and J. R. Barker, J. Chem. Phys. 74, 3823 (1981).

${ }^{13}$ (a) T. Shimanouchi, Tables of Molecular Vibrational Frequencies, Natl. Stand. Ref. Data Serv. Natl. Bur. Stand. 39 (U.S. GPO, Washington, D.C., 1972), Vol. 1; 39, (1972); (b) T. Shimanouchi, J. Phys. Chem. Ref. Data 6, 993 (1977).

${ }^{14}$ A. C. Baldwin and J. R. Barker, J. Chem. Phys. 74, 3813 (1981).

${ }^{15}$ D. T. Gillespie, J. Comput. Phys. 22, 403 (1976); J. Phys. 81, 2340 (1977); J. Comput. Phys. 28, 395 (1978).

${ }^{16}$ J. R. Barker, J. Chem. Phys. 72, 3686 (1980).

${ }^{17}$ S. E. Stein and B. S. Rabinovitch, J. Chem. Phys. 58, 2438 (1973).

${ }^{18}$ G. Z. Whitten and B. S. Rabinovitch, J. Chem. Phys. 38, 2466 (1963); 41, 1883 (1964)

${ }^{19}$ (a) M. Quack, J. Chem. Phys. 69, 1282 (1978); (b) Chem. Phys. Lett. 65 , 140 (1979); (c) Ber. Bunsenges. Phys. Chem. 83, 757 (1979); (d) 85, 318 (1981); Dynamics of the Excited State, edited by K. P. Lawley (Wiley, New York, 1982), 395.

${ }^{20}$ A. C. Baldwin and J. R. Barker, J. Chem. Phys. 74, 3823 (1981).

${ }^{21}$ J.-M. Zellweger, T. C. Brown, and J. R. Barker, J. Phys. Chem. (to be published).

${ }^{22}$ For detailed treatments of heterogeneous deactivation and mass transport to the cell walls in other systems, see (a) R. G. Gilbert, T. T. Nguyen, and K. D. King, Int. J. Chem. Kinet. 11, 317 (1979); (b) R. G. Gilbert and K. D. King, Chem. Phys. 49, 367 (1980).

${ }^{23}$ T. C. Brown, K. D. King, J.-M. Zellweger, and J. R. Barker, Ber. Bunsenges. Phys. Chem. 89, 301 (1985). 